\title{
Introduction to the Workshop
}

Mark P. Bridgen

I nstructors of classes in plant breeding are always looking for new laboratory exercises to enhance their students' learning experiences. However, plant breeding laboratory exercises are often difficult to compress into one semester; the development of new exercises can be unsuccessful and time-consuming; and information on plant breeding is difficult to locate. In addition, instructors have to consider the expectations of administrators, teaching assistants, and students. Instructors want laboratories that are reliable, can be completed within one semester, meet objectives valuable to the course, familiarize students with specific skills, have sufficient background information, and do not have lengthy planning and preparation. Administrators would like exercises to be inexpensive. Teaching assistants prefer laboratories that are implemented easily and outlined in detail to decrease confusion and keep laboratory reports short. Finally, the students want laboratories that are valuable, easy to follow, and described clearly and concisely.

During the 1998 ASHS Annual Conference, the Ornamental Plant Breeding Working Group, sponsored a workshop titled Laboratory Techniques for Teaching Plant Breeding. The objective of the workshop was to describe laboratory exercises that can be used in plant breeding classes. Four seasoned instructors and authorities of plant breeding participated in the workshop. The speakers outlined four exercises that can be easily implemented into any plant breeding class. The laboratory exercises that were described included the use of Wisconsin Fast Plants for plant breeding, a segregation exercise for flower color, molecular techniques for plant breeding, and techniques for ploidy determination.

Irwin Goldman from the University of Wisconsin presents his paper on the Teaching Recurrent Selection in the Classroom with Wisconsin Fast Plants. This laboratory allows a breeding project on selection to be completed in one semester by using the Wisconsin Fast Plants. This Brassica species has such a short life cycle that it is easy to use for genetic studies. The cyclical selection process of recurrent selection can easily be taught with the Fast Plants because they allow three cycles of recurrent selection during a 15-week semester. This gives the students experiences in selection, pollination, and seed harvest while demonstrating phenotypic changes through selection.

Chiwon Lee, from North Dakota State University, gives a unique and interesting explanation of segregation in his Laboratory Exercise on the Segregation of Flower Color and Related Genes Using Velvet Flower (Salpiglossis sinuata Ruiz and Pavon). By using velvet flower, a plant easy to grow in the greenhouse, the students can learn about pigmentation patterns. In just 3 weeks after self- or cross- pollination, seeds can be collected. Then, the influences of three genes that control flower color and pigmentation patterns can be conveniently demonstrated with their dominant and recessive alleles. Other exercises in plant breeding, such as the induction and use or polyploid plants in plant breeding, can be demonstrated with this species.

The presentation by Richard Veilleux from the Virginia Polytechnic Institute and State University on Anther Culture of Potato and Molecular Analysis of Anther-derived Plants as Laboratory Exercises for Plant Breeding Courses gives the students a molecular experience with plant breeding as well as an understanding of haploid plants. In just 5 weeks, androgenic embryos can be obtained in a classroom, and a month later, be large enough for ploidy determination by chromosome counts in root tips, chloroplast counts in stomatal guard cells, or flow cytometry of nuclei released from in vitro plantlets. Deoxyribonucleic Acid (DNA) can be extracted from anther-derived plantlets using a rapid extraction procedure to demonstrate segregation of Polymerase Chain Reaction (PCR)-based markers such as randomly amplified polymorphic DNA (RAPD) markers, randomly amplified microsatellite polymorphisms (RAMPs) or microsatellites. Microsatellite markers that were heterozygous in the anther donor can be used to verify haploidy in anther-derived plants. By proper planning and scheduling, these exercises can all be completed in one semester.

Christopher Cramer, from New Mexico State University, presents a great and comprehensive exercise on Laboratory Techniquesfor Determining Ploidy in Plants. Experiences with root tip squashes, pollen mother cell squashes, pollen grain size and germinal pore counts, stomata size and density determination, and gross morphology are all possible by following the procedures outlined in this paper. By the end of the semester, students are able to determine ploidy levels in plants and understand how to recognize the steps required to determine ploidy level. This is a good exercise to get students to think and to plan a strategy for plant breeding.

Professor, Ornamental Plant Breeding Working Group, Chair, Department of Plant Science, U-67, 1376 Storrs Road, University of Connecticut, Storrs, CT 06269.

The cost of publishing this paper was defrayed in part by the payment of page charges. Under postal regulations, this paper therefore must be hereby marked advertisement solely to indicate this fact. 